\title{
The first total laparoscopic pancreatoduodenectomy in Poland
}

\author{
Andrzej Budzyński ${ }^{1}$, Anna Zub-Pokrowiecka ${ }^{1}$, Anna Zychowicz ${ }^{1}$, Michał Pędziwiatr ${ }^{1}$, Mateusz Wierdak ${ }^{2}$, Maciej Matłok ${ }^{1}$, \\ Małgorzata Zając ${ }^{1}$ \\ ${ }^{1} 2^{\text {nd }}$ Department of General Surgery, Department of Endoscopic, Metabolic and Soft Tissue Tumors Surgery, Jagiellonian University \\ Medical College, Krakow, Poland \\ 2Department of Physiology, Jagiellonian University Medical College, Krakow, Poland
}

Videosurgery Miniinv 2014; 9 (3): 453-457

DOI: $10.5114 /$ wiitm.2014.45034

\begin{abstract}
We present a case of a 55-year-old female patient with pancreatic head cancer who was treated with total laparoscopic pylorus-preserving pancreatoduodenectomy (TLPD) on 13.12.2013. The procedure as well as the postoperative course was uncomplicated. The patient was mobilized on the day of surgery; a liquid diet was introduced on day 1 and a full hospital diet on day 2 postoperatively. Drains were removed on the $3^{\text {rd }}$ day after the procedure. Length of hospital stay was 6 days. The final pathology report confirmed the diagnosis of cancer. According to our knowledge this is the first report on total laparoscopic pancreatoduodenectomy in Poland performed by an entirely Polish team of surgeons. In our opinion, TLPD is feasible and similarly to other laparoscopic operations may improve postoperative recovery.
\end{abstract}

Key words: laparoscopy, fast-track, enhanced recovery, pancreatoduodenectomy, Whipple, pancreatic cancer.

\section{Introduction}

Michel Gagner was the first to describe total laparoscopic pancreatoduodenectomy (TLPD), in 1994 [1]. Minimally invasive operations in patients with pancreatic tumors have proven to be among the most demanding and complicated laparoscopic procedures. Thus in some authors' opinion, they should be performed only by very experienced surgeons in high volume pancreatic centers [2]. Although almost 20 years have passed since the first TLPD by Gagner, there has not been much acceptance of this technique to date. Some authors suggest limiting the use of laparoscopy in pancreatic head cancer only to staging $[3,4]$. According to recent studies only a few hundred patients have undergone TLPD worldwide [5]. Despite longer operation time, minimally invasive procedures are associated with reduced blood loss, faster recovery and shorter hospital stay [6]. On Friday, $13^{\text {th }}$ December 2013 we successfully attempted a totally laparoscopic pylorus preserving pancreatoduodenectomy in a patient with pancreatic head cancer. According to our knowledge it is the first report of a TLPD performed by an entirely Polish team of surgeons.

\section{Case report}

A 55 -year-old female patient $(158 \mathrm{~cm}, 58 \mathrm{~kg}$, body mass index (BMI) $23.2 \mathrm{~kg} / \mathrm{m}^{2}$ ), an active smoker, was admitted with symptoms of obstructive jaundice. She complained of epigastric pain lasting for the last month. She was in a good general condition with no comorbidities. The patient had undergone open appendectomy and two cesarean sections some years before. The family history was unremarkable.

\section{Address for correspondence}

Michał Pędziwiatr MD, PhD, Department of Endoscopic, Metabolic and Soft Tissue Tumors Surgery, University Hospital, 21 Kopernika St, 31-416 Krakow, Poland, phone: +48 608552 323, e-mail: mpedziwiatr@gmail.com 
On physical examination she presented with jaundice and was otherwise asymptomatic. Abdominal ultrasound revealed cholestasis and an irregular hypoechoic mass $3 \mathrm{~cm}$ in diameter in the head of the pancreas. In endoscopic retrograde cholangiopancreatography (ERCP) there was a narrowing of the distal common bile duct. It was not conclusive but highly suspicious for pancreatic head adenocarcinoma. Abdominal and pelvic magnetic resonance imaging (MRI) confirmed the diagnosis and excluded metastatic dissemination. Ca 19-9 and CEA markers were in the normal range. The patient was scheduled for surgery and ten days later laparoscopic pylorus preserving pancreatoduodenectomy was successfully performed.

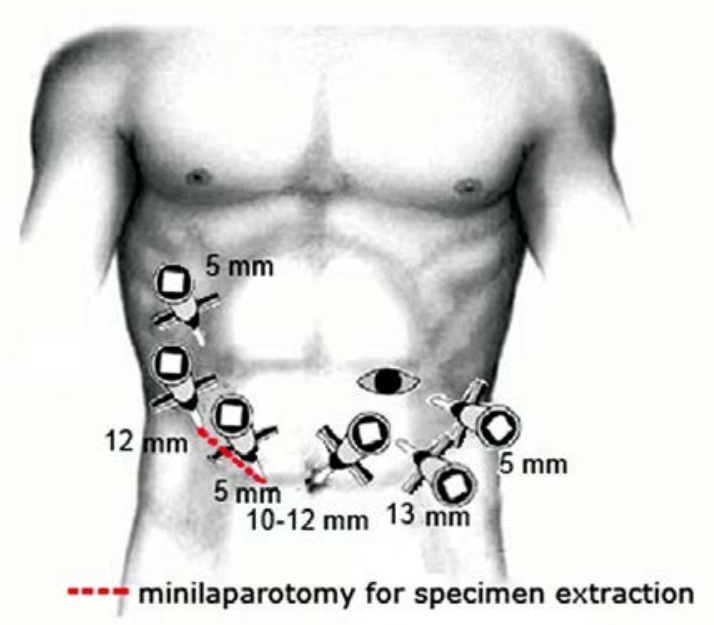

Figure 1. Trocar placement for TLPD

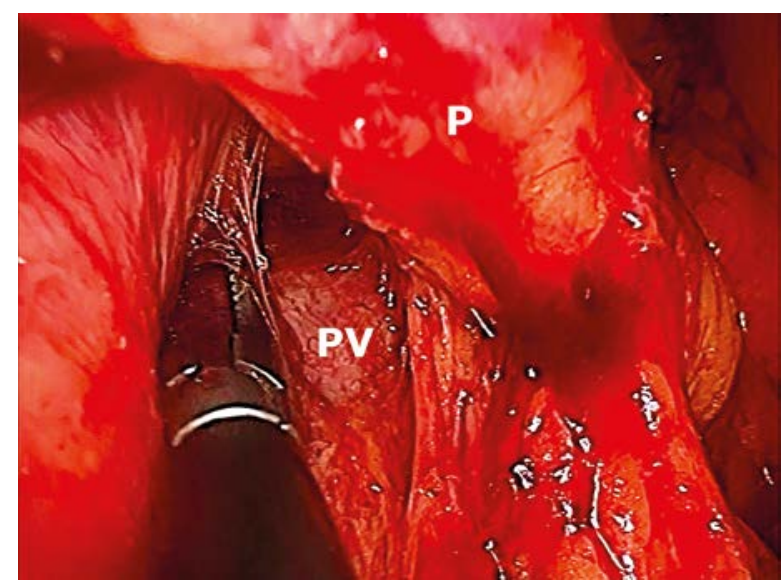

Photo 1. Dissection of the neck of the pancreas from the portal vein

$P$ - pancreas, $P V-$ portal vein

\section{Operative technique}

The patient was in the supine position. After establishing pneumoperitoneum a total of 6 (5-12 $\mathrm{mm})$ trocars were placed in a semicircle as shown in Figure 1. Exploratory laparoscopy did not reveal metastatic spread or ascites. After division of the gastrocolic ligament the lesser sac was widely exposed. The right flexure of the colon was mobilized and a Kocher maneuver was performed. Hepatic common, hepatic proper arteries and common bile duct were identified. The neck of the pancreas was dissected from the portal vein confluence (Photo 1). In the absence of neoplastic infiltration of major vessels, gastroduodenal and right gastric arteries were clipped and divided. The neck of the pancreas was divided with a harmonic scalpel. The first portion of the duodenum was transected with an Echelon stapler 2-3 cm distal to the pylorus. In the next step cholecystectomy from the fundus was performed and the common hepatic duct was identified (Photo 2) and transected at the level of the cystic duct. The third and fourth portion of the duodenum and proximal jejunum were mobilized and passed underneath the superior mesenteric vessels. The head of the pancreas with the tumor was dissected from superior mesenteric vessels using Ligasure. Larger arterial and venous branches including the inferior pancreaticoduodenal artery were clipped and divided. An additional lymphadenectomy of group 8 (located around the common hepatic artery) and group 12 (located around the hepatic proper artery) lymph nodes was performed. Before anastomoses were fin-

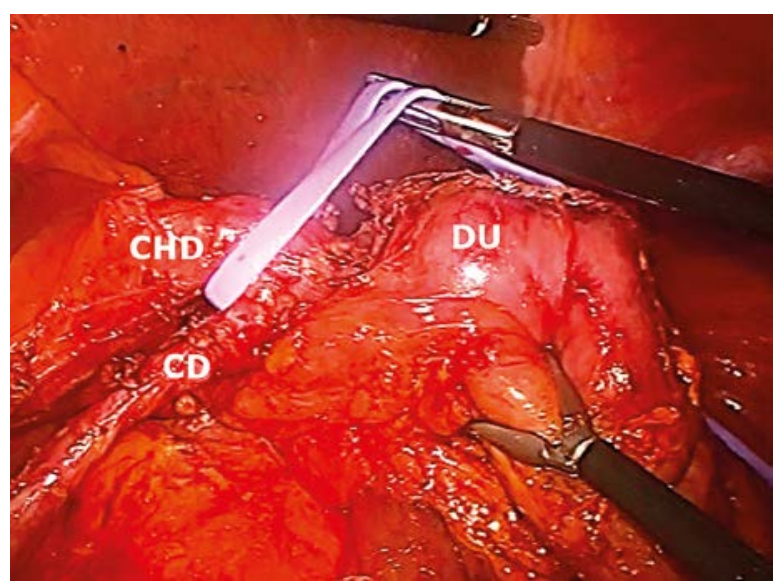

Photo 2. Common hepatic duct ready to transect

CHD - common hepatic duct, $C D$ - cystic duct, $D U$ - duodenum 
ished the specimen in one piece consisting of the pancreatic head with the tumor, duodenum and the gallbladder was placed in a plastic bag and at the end removed via infraumbilical minilaparotomy additionally protected with a plastic sleeve to separate it from the specimen (Photo 3). The proximal jejunum was passed through the mesocolon. A plastic stent $5 \mathrm{Fr} / 15 \mathrm{~cm}$ was inserted into the pancreatic duct and an end-to-end pancreaticojejunostomy was performed (2 layers - inner layer with running absorbable 3-0 Vicryl and outer layer of interrupted covering 3-0 Vicryl) (Photos 4 and 5). Next, end-toside hepaticojejunostomy with continuous 4-0 Vicryl was performed (Photo 6). Finally the continuity of the digestive tract was restored with an end-to-side duodenojejunostomy with 2 layers of running Vic-

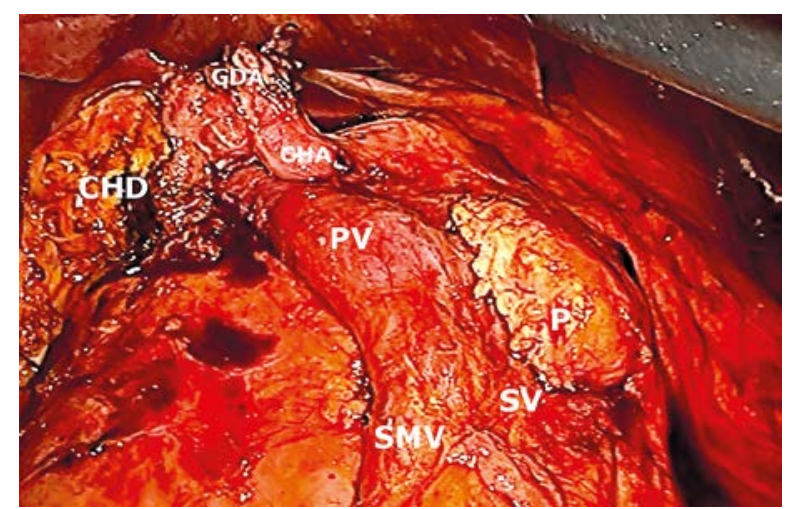

Photo 3. Operating field after specimen extraction

CHD - common hepatic duct, GDA - gastroduodenal artery (clip ped), CHA - common hepatic artery, PV-portal vein, SMV - superior mesenteric vein, $S V$ - splenic vein, $P$ - pancreas

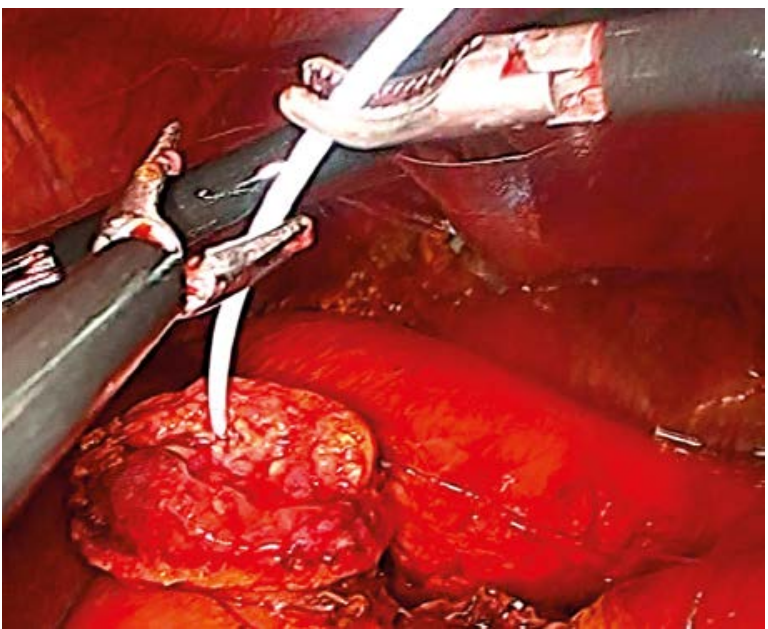

Photo 5. Stenting the pancreatic duct ryl 3-0. After meticulous hemostasis 2 drains were placed near the pancreaticojejunostomy and hepaticojejunostomy. Wounds were sutured with absorbable sutures.

\section{Postoperative care}

After anesthesia the patient was transferred to the recovery room where she spent a few hours before admittance to the general ward. The urinary catheter was removed before leaving the recovery room. Postoperative analgesia was based on an epidural catheter in combination with intravenous/ oral non-steroid anti-inflammatory drugs and paracetamol. Low doses of morphine were necessary only for the first few hours in the recovery room. On the day of surgery she was allowed to drink a few

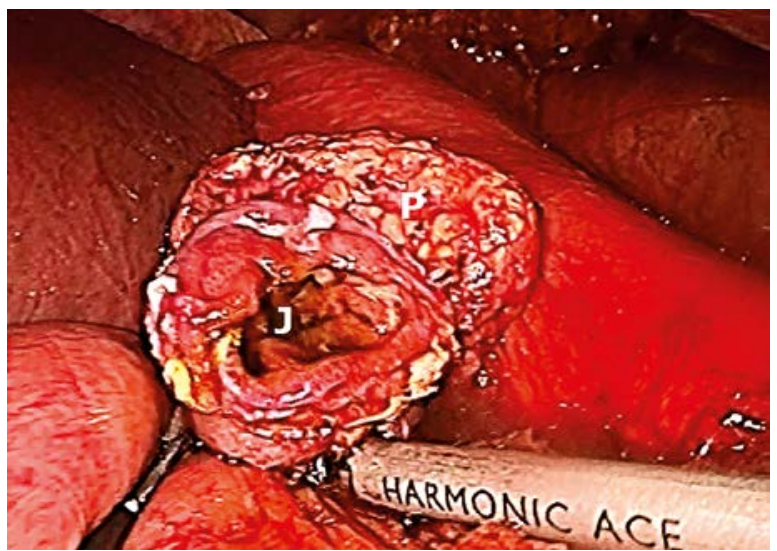

Photo 4. Outer layer of end-to-end pancreaticojejunostomy

$J$-jejunum, $P$ - pancreas

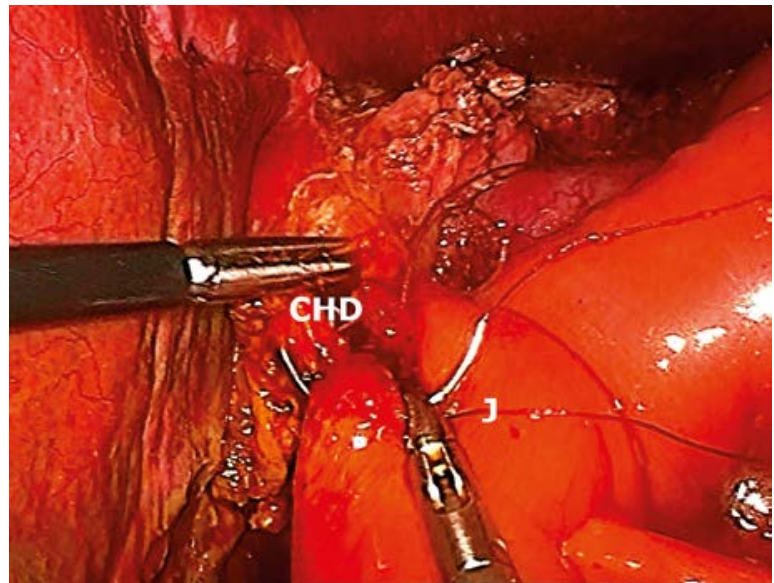

Photo 6. End-to-side hepaticojejunostomy

CHD - common hepatic duct, J - jejunum 
sips of clear water. On the $1^{\text {st }}$ postoperative day the patient was allowed to drink, and on the following day a liquid diet (protein-rich oral supplements) was introduced. A full diet was begun on the $3^{\text {rd }}$ postoperative day. She was mobilized out of bed on the $1^{\text {st }}$ postoperative day and was freely walking without assistance on the following day. Intravenous fluids were stopped on the $2^{\text {nd }}$ postoperative day when oral fluid intake was sufficient. Drains were removed on the $3^{\text {rd }}$ postoperative day. There were no perioperative complications. She was discharged on the $6^{\text {th }}$ day of hospital stay fully mobilized, with complete tolerance for an oral diet and with no need for any analgesia or other medications.

\section{Discussion}

The advantages of a minimally invasive approach to pancreatic surgery are well documented for distal pancreatectomies with evidence of faster recovery, reduced blood loss, a lower complications rate and shorter hospital stay [7-9]. The main parameters in quality of oncological operations are radical resection, complication rate, postoperative recovery (length of stay), quality of life and survival. Although there are no randomized trials comparing open and laparoscopic pancreatoduodenectomy, initial reports about minimally invasive operations are very encouraging [10]. We chose to perform a pylorus-preserving procedure as it is our method of choice in open surgery which is associated with decreased operating times, fewer blood transfusions, lower mortality and improved long-term patient survival compared to a typical Whipple procedure [11]. Results from laparoscopic pancreatic centers show that TLPD operation time is longer. However, Kendrick assumes that most surgeons are within the initial learning curve and the operation time can be reduced. He noted a decrease of operative time from a mean of $7.7 \mathrm{~h}$ in the first 10 patients to $5.3 \mathrm{~h}$ in the last 10 patients treated in Mayo Clinic [4]. Operative time of the TLPD we performed was $480 \mathrm{~min}$, and it is comparable to other authors' reports [6]. There were no perioperative complications. It is however well established that pancreatic surgery is associated with a significant rate of early complications. The most significant cause of perioperative morbidity is pancreatic fistula, observed in up to $20 \%$ cases [12]. A meta-analysis of 150 TLPD procedures revealed that the fistula rate is comparable with those performed with an open technique [6]. We can also assume (although there is not yet strong evidence) that the overall complication rate (especially wound infection or incisional hernia) will decrease, similarly to other laparoscopic procedures. One can also expect faster recovery after surgery. Perioperative care in the presented patient was based on ERAS Society Guidelines for patients undergoing pancreatoduodenectomy [13]. Length of stay was 6 days. Oral fluids were introduced on the day of surgery; a soft diet was introduced on the next day, and a full diet on the second postoperative day. It allowed us to reduce intravenous fluids and shortened the hospital stay. The benefits of ERAS protocols are well documented in other studies, and it seems that the advantages of laparoscopy would improve recovery and reduce perioperative complications $[14,15]$. The biggest concern is still the long-term oncological outcome of the laparoscopic technique. The pathology result of the removed specimen confirmed the presence of pancreatic adenocarcinoma G2 (pT3N1). There are no studies investigating long-term results after TLPD. Some authors have pointed out that the $\mathrm{RO}$ resection rate and lymph node harvest in TLPD are similar to open procedures, and especially that extended lymphadenectomy does not improve survival $[3,4,16]$. This suggests that if laparoscopy does not improve outcomes, the quality of specimens guarantees the possibility of noninferiority. There is only one report of a port site metastasis after TLPD, which after so many procedures being performed so far does not seem to be a significant problem [17].

\section{Conclusions}

We consider total laparoscopic pylorus-preserving pancreatoduodenectomy safe and feasible. The TLPD procedure we present is according to our knowledge the one in Poland. The laparoscopic approach has advantages and drawbacks typical for minimally invasive operations. It is difficult to estimate treatment outcomes after 1 case, but we can expect smaller postoperative trauma and faster recovery. The current literature and lack of randomized trials is insufficient to demonstrate the advantages of laparoscopic over open pancreatoduodenectomy in terms of complication rate and long-term outcomes. Although the preliminary results are promising, further studies on this matter should be conducted. 


\section{Acknowledgments}

The publication of this article was supported by Faculty of Medicine, Jagiellonian University Medical College, Leading National Research Centre (KNOW) 2012-2017.

\section{References}

1. Gagner M, Pomp A, Laparoscopic pylorus-preserving pancreatoduodenectomy. Surg Endosc 1994; 8: 408-10,

2. Dulucq JL, Wintringer P, Stabilini C, et al. Are major laparoscopic pancreatic resections worthwhile? A prospective study of 32 patients in a single institution. Surg Endosc 2005; 19: 1028-34.

3. Gagner M, Palermo M. Laparoscopic Whipple procedure: review of the literature. J Hepatobiliary Pancreat Surg 2009; 16: 726-30.

4. Kendrick ML, Cusati D. Total laparoscopic pancreaticoduodenectomy: feasibility and outcome in an early experience. Arch Surg 2010; 145: 19-23.

5. Gumbs AA, Rodriguez Rivera AM, Milone L, et al. Laparoscopic pancreatoduodenectomy: a review of 285 published cases. Ann Surg Oncol 2011; 18: 1335-41.

6. Jacobs MJ, Kamyab A. Total laparoscopic pancreaticoduodenectomy. JSLS 2013; 17: 188-93.

7. Kooby DA, Gillespie T, Bentrem D, et al. Left-sided pancreatec tomy: a multicenter comparison of laparoscopic and open approaches. Ann Surg 2008; 248: 438-46.

8. Costi R, Randone B, Mal F, et al. Laparoscopic minor pancreatic resections (enucleations/atypical resections). A long-term appraisal of a supposed mini-invasive approach. Videosurgery Miniinv 2013; 8: 117-29.

9. Michalik M, Bobowicz M, Lech M, et al. Distal pancreatic resection via laparo-endoscopic single site surgery - development of the technique. Videosurgery Miniinv 2010; 4: 142-5.

10. Palanivelu C, Jani K, Senthilnathan P, et al. Laparoscopic pancreaticoduodenectomy: technique and outcomes. J Am Coll Surg 2007; 205: 222-30.

11. Wenger FA, Jacobi CA, Haubold K, et al. Gastrointestinal quality of life after duodenopancreatectomy in pancreatic carcinoma. Preliminary results of a prospective randomized study: pancreatoduodenectomy or pylorus-preserving pancreatoduodenectomy. Chirurg 1999; 70: 1454-9.

12. Stojadinovic A, Brooks A, Hoos A, et al. An evidence-based approach to the surgical management of resectable pancreatic adenocarcinoma. J Am Coll Surg 2003; 196: 954-64.

13. Lassen K, Coolsen MM, Slim K, et al. Guidelines for perioperative care for pancreaticoduodenectomy: Enhanced Recovery After Surgery (ERAS(R)) Society recommendations. World J Surg 2013; 37: 240-58.

14. Robertson N, Gallacher PJ, Peel N, et al. Implementation of an enhanced recovery programme following pancreaticoduodenectomy. HPB (Oxford) 2012; 14: 700-8.

15. Abu Hilal M, Di Fabio F, Badran A, et al. Implementation of enhanced recovery programme after pancreatoduodenectomy: a single-centre UK pilot study. Pancreatology 2013; 13: 58-62.

16. Zheng MH, Feng B, Lu AG, et al. Laparoscopic pancreaticoduodenectomy for ductal adenocarcinoma of common bile duct: a case report and literature review. Med Sci Monit 2006; 12: CS57-60.

17. Young S, Abbitt P, Hughes SJ. Port-site recurrence of pancreatic adenocarcinoma following laparoscopic pancreaticoduodenectomy. J Gastrointest Surg 2012; 16: 2294-6.

Received: 24.01.2014, accepted: 9.03.2014. 